\title{
Using OOK Modulation for Symmetric 40-Gb/s Long-Reach Time-Sharing Passive Optical Networks
}

\author{
Chien-Hung Yeh, Chi-Wai Chow, Chia-Hsuan Wang, Yu-Fu Wu, Fu-Yuan Shih, and Sien Chi
}

\begin{abstract}
Due to the requirement of broad bandwidth for next-generation access networks, present passive optical networks (PONs) will be upgraded to $40 \mathrm{~Gb} / \mathrm{s}$ or higher data rate PONs. Hence, we propose and experimentally demonstrate a simple and efficient scheme to achieve a symmetric $40-\mathrm{Gb} / \mathrm{s}$ long-reach (LR) time-division-multiplexed PON by using four wavelength-division-multiplexed 10-Gb/s external on-off keying format channels to serve as the optical transmitter for downstream and upstream traffic simultaneously. Moreover, the system performance of $L R$ transmission and split ratio have also been analyzed and discussed without dispersion compensation.
\end{abstract}

Index Terms-40 Gb/s, long reach (LR), passive optical network (PON), time-division multiplexing (TDM).

\section{INTRODUCTION}

$\mathbf{T}$ HE bandwidth demand for broadband services, such as the high-definition Internet protocol television (HD-IPTV), digital cinema, tele-presence, online gaming, etc., will make the future access networks supporting higher and higher data rates. Thus, passive optical network (PON) architecture would be the best choice for the last mile access due to the benefits of high-capacity and cost-effectiveness. Several time-division-multiplexed PON (TDM-PON) architectures have been developed and standardized, such as the broadband PON (BPON), Ethernet PON (EPON), and gigabit PON (GPON) [1]-[3]. And these PONs have already been deployed by Internet service providers (ISPs) today [4]-[6].

Moreover, the bandwidth of standardized $10-\mathrm{Gb} / \mathrm{s}$ TDM-PONs may not be large enough to meet the continually-increasing bandwidth demand for future multimedia services [7]-[9]. Therefore, next-generation PONs will be supporting data rates of up to $40 \mathrm{~Gb} / \mathrm{s}$ or higher. Using a single channel of $40-\mathrm{Gb} / \mathrm{s}$ data rate for transmission systems could be possible. However, the $40-\mathrm{Gb} / \mathrm{s}$ data rate signal will limit the transmission distance in the optical network to within a few kilometers by using on-off keying (OOK) modulation due to

Manuscript received October 12, 2009; revised February 04, 2010; accepted February 09, 2010. Date of publication February 22, 2010; date of current version April 02, 2010. This work was supported by the National Science Council, Taiwan, under Contract NSC-98-2622-E-009-185-CC2, Contract NSC-98-2221-E-009-017-MY3, and Contract NSC-97-2221-E-009-038-MY3.

C.-H. Yeh is with the Information and Communications Research Laboratories (ICL), Industrial Technology Research Institute (ITRI), Chutung, Hsinchu 31040, Taiwan (e-mail: depew@itri.org.tw).

C.-W. Chow, C.-H. Wang, Y.-F. Wu, F.-Y. Shih, and S. Chi are with the Department of Photonics and Institute of Electro-Optical Engineering, National Chiao Tung University, Hsinchu 30010, Taiwan.

Color versions of one or more of the figures in this letter are available online at http://ieeexplore.ieee.org.

Digital Object Identifier 10.1109/LPT.2010.2043353 the fiber chromatic dispersion. In addition, the unavailability of the commercial $40-\mathrm{Gb} / \mathrm{s}$ burst-mode receiver $(\mathrm{Rx})$ is also one of the major restrictions to deploying the 40-Gb/s TDM-PON system. Furthermore, to reduce the cost of the future fiber access networks, long-reach (LR) PONs have also been proposed and studied [10]-[13].

Here we propose and investigate a simple and efficient architecture to achieve 40-Gb/s LR TDM-PON deployment using four wavelength-division-multiplexed (WDM) 10-Gb/s external modulated distributed-feedback laser diodes (DFB-LDs) acting as the optical transmitters for downstream and upstream directions. As a result, a $40-\mathrm{Gb} / \mathrm{s}$ traffic can be efficiently achieved without changing the network infrastructure. In addition, the proposed $40-\mathrm{Gb} / \mathrm{s}$ LR TDM-PON requires a moderate upgrade of components at the optical networking unit (ONU) and the optical line terminal (OLT). The synchronization of these four externally modulated DFB-LDs and the four burst-mode Rxs is performed in the media access controller (MAC) layer. Using external modulation and optical amplifiers could increase the deployment cost of the LR-PON. However, these LR-PONs would replace the separate metro and access networks with a single and integrated system. This approach is expected to produce significant capital and operational cost savings, since the number of network elements and interfaces can be reduced, together with the design complexity and power consumption [13].

\section{EXPERIMENT AND DISCUSSION}

The experimental setup of the proposed $40-\mathrm{Gb} / \mathrm{s}$ LR TDM-PON is illustrated in Fig. 1. At the OLT, the $40-\mathrm{Gb} / \mathrm{s}$ downstream signal is achieved by using four WDM DFB-LDs with $10-\mathrm{Gb} / \mathrm{s}$ external OOK modulation. In the experiment, a Mach-Zehnder modulator (MZM) was used. As shown in Fig. 1, the four DFB-LDs $\left(\mathrm{LD}_{1}\right.$ to $\left.\mathrm{LD}_{4}\right)$ are multiplexed by a $4 \times 1$ wavelength-division multiplexer (WDM) with insertion loss of $5 \mathrm{~dB}$. The downstream traffic is broadcasting through a three-port optical circulator (OC) and 80-km standard single-mode fiber (SMF) to the remote node (RN). In the RN, a bidirectional erbium-doped fiber amplifier (EDFA) is used to compensate the insertion losses of other components and a $1 \times N$ optical splitter (SP) is used for broadcasting downstream information to ONUs. In each ONU, the upstream signals are also produced by four WDM DFB-LDs with $10-\mathrm{Gb} / \mathrm{s}$ OOK external modulation to achieve $40 \mathrm{~Gb} / \mathrm{s}$. Besides, the inset of Fig. 1 shows the wavelength arranging for the downstream and upstream channels. 


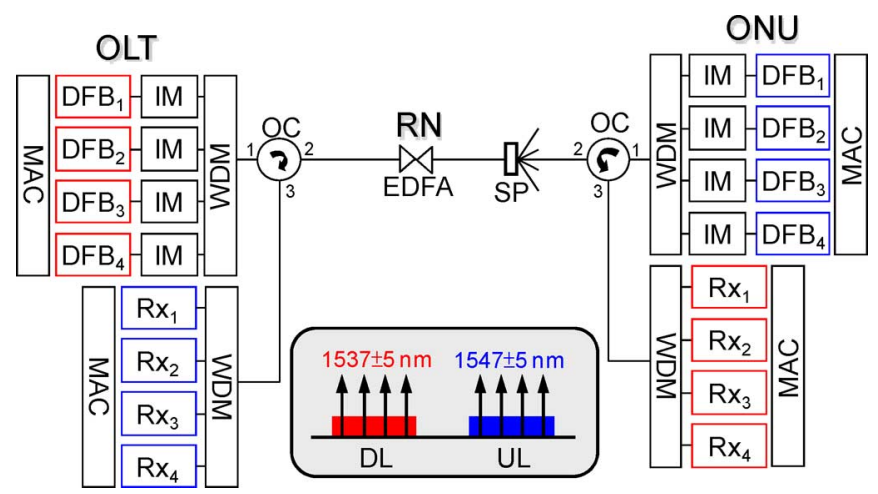

Fig. 1. Proposed LR TDM-PON architecture. DFB: distributed-feedback laser diode; IM: intensity modulator; WDM: wavelength-division multiplexer; SMF: single-mode fiber; RN: remote node; MAC: media access control; ONU: optical networking unit; Rx: receiver.



Fig. 2. Measured optical spectra of four downstream channels (from 1535 to $1538 \mathrm{~nm}$ ) and upstream channels (from 1545 to $1548 \mathrm{~nm}$ ), respectively.

Fig. 2 shows the optical spectra of downstream and upstream wavelengths which are measured by an optical spectrum analyzer (OSA) with a resolution of $0.01 \mathrm{~nm}$. In the experiment, four WDM wavelengths at $1535,1536,1537$, and $1538 \mathrm{~nm}$ with output powers of about $3,2.9,2.7$, and $2.7 \mathrm{dBm}$, respectively, are used for downstream channels. And another four wavelengths at $1545,1546,1547$, and $1548 \mathrm{~nm}$, with output powers of about $2.7,2.8,3.1$, and $3 \mathrm{dBm}$, respectively, are used for upstream signals. Each DFB-LD is modulated at $10-\mathrm{Gb} / \mathrm{s}$ nonreturn-to-zero (NRZ) format, with a pseudorandom binary sequence (PRBS) of $2^{31}-1$ by intensity modulator (IM).

In the 40-Gb/s TDM access network, a single channel with $40-\mathrm{Gb} / \mathrm{s}$ data rate could be used. In accordance with the present PON standards [1]-[3], the maximum transmission length is $20 \mathrm{~km}$. However, the transmission of $40-\mathrm{Gb} / \mathrm{s}$ OOK signal would be limited by fiber chromatic dispersion. Here, we use commercial software (VPI Transmission Maker V7.5) to numerically analyze the $40-\mathrm{Gb} / \mathrm{s}$ OOK and differential phase-shift keying (DPSK) downstream signals in the PON. In the simulation, both signals are at $1545 \mathrm{~nm}$, average output power of $0 \mathrm{dBm}$. The OOK and DPSK signals are generated by an MZM and a phase modulator, respectively. Both signals are launched into different lengths of SMF (dispersion parameter $=17 \mathrm{ps} / \mathrm{nm} / \mathrm{km}$ ). The DPSK is demodulated by using a 1-bit delay interferometer and is detected using single-end. Fig. 3(a) shows the bit-error-rate (BER) performance at back-to-back (B2B), 5- and 10-km
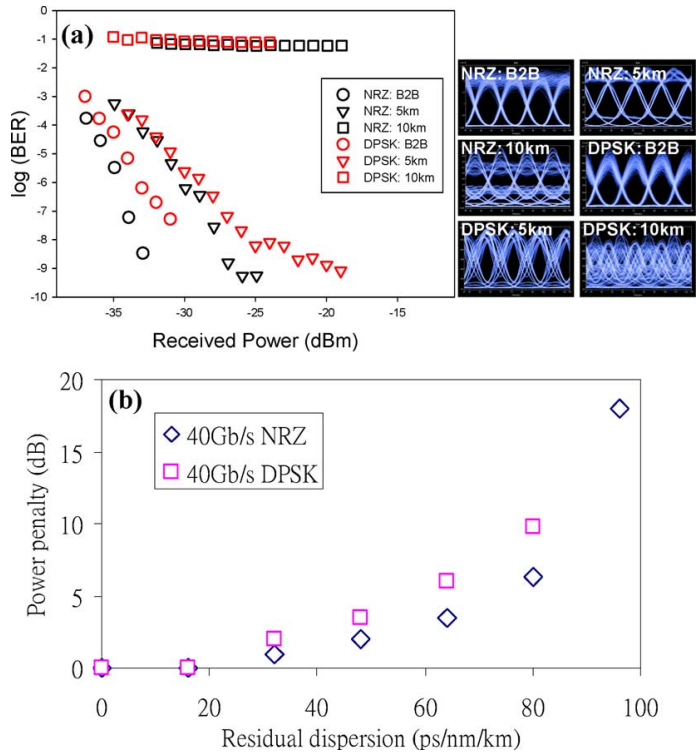

Fig. 3. (a) Numerical analysis of BER performance at $40-\mathrm{Gb} / \mathrm{s}$ NRZ and DPSK modulation formats under $\mathrm{B} 2 \mathrm{~B}, 5-$, and $10-\mathrm{km}$ transmission, respectively. (b) Received power penalty under different residual dispersion at $40-\mathrm{Gb} / \mathrm{s} \mathrm{NRZ}$ and DPSK modulation formats. The insets are the corresponding eye diagrams.

transmission, respectively. We can observe the power penalties of 6.3 and $9.8 \mathrm{~dB}$ at the BER of $10^{-9}$ of OOK and DPSK in $5-\mathrm{km}$ transmission due to fiber chromatic dispersion. When the transmission extends to $10 \mathrm{~km}$, the BER almost cannot be measured, as also seen in Fig. 3(a). The insets of Fig. 3(a) show the corresponding eye diagrams. We also studied the case when dispersion compensation is used. In the simulation, a $20-\mathrm{km}$ standard SMF was dispersion compensated by dispersion-compensating fiber (DCF). However, exact dispersion compensation never occurs due to the temperature changes and/or different dispersion slopes of the SMF and DCF. Fig. 3(b) shows that power penalty of $\sim 2 \mathrm{~dB}$ was observed in the NRZ signal when residual dispersion was $48 \mathrm{ps} / \mathrm{nm} / \mathrm{km}$ ( $\sim 3 \mathrm{~km}$ of SMF). As a result, in order to overcome the fiber dispersion at higher data rates, we could employ four WDM channels with $10 \mathrm{~Gb} / \mathrm{s}$ to perform the $40-\mathrm{Gb} / \mathrm{s}$ transmission in TDM-PON.

In the experimental BER measurement of our proposed LR-PON, we use a $1545-\mathrm{nm}$ wavelength to represent the data traffic. Fig. 4 presents the BER performance under B2B, 75and $100-\mathrm{km}$ transmission lengths, respectively. As shown in Fig. 4, error-free operation can be achieved when the transmission distance is up to $100 \mathrm{~km}$. The $3-$ and $4-\mathrm{dB}$ power penalties were observed after $75-$ and $100-\mathrm{km}$ transmission of SMF, respectively. The power penalties were due to the dispersion of the SMF since no dispersion compensation was used. The corresponding eye diagrams are also shown in the insets of Fig. 4.

Signal splitting capability is one important issue in a TDM-PON. Here, the split-ratio of our proposed scheme is also investigated. Fig. 5 shows the BER performances of $10-\mathrm{Gb} / \mathrm{s}$ NRZ data at wavelength $1545 \mathrm{~nm}$ with PRBS $2^{31}-1$ with different split-ratio in the transmission link of 75 and $100 \mathrm{~km}$ without any dispersion compensation. The corresponding eye diagrams are shown in the insets of Fig. 5. According to the 




Fig. 4. BERs versus received optical power of the proposed $40-\mathrm{Gb} / \mathrm{s}$ TDM-PON with B2B, 75-, and 100-km SMF transmission. Insets: the corresponding eye diagrams of B2B, 75-, and 100-km SMF transmission at 1545-nm wavelength.

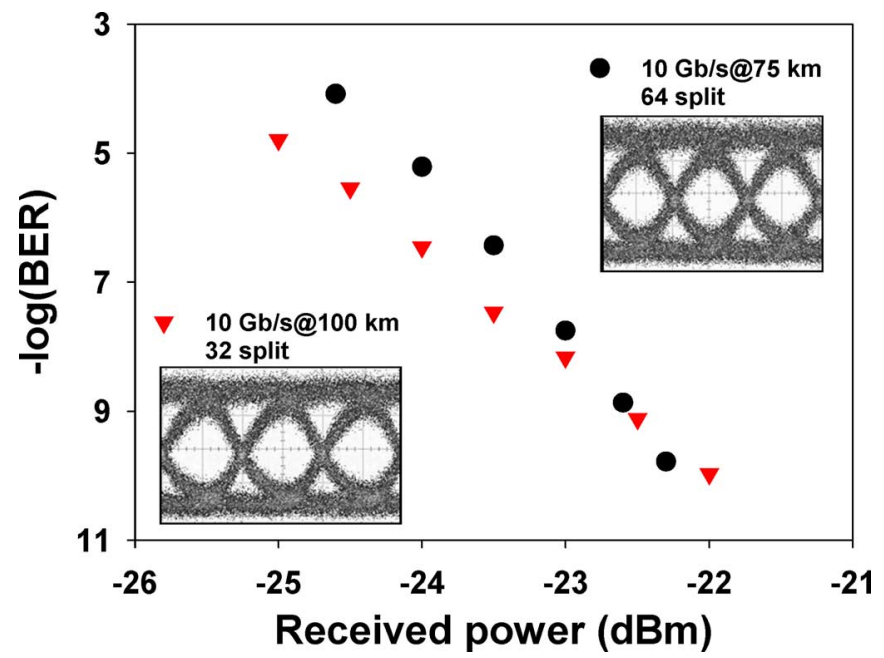

Fig. 5. BER performances of 10-Gb/s NRZ signals with 64- and 32-split after 75- and 100-km SMF transmission link, respectively. Insets show the corresponding eye diagrams.

proposed PON system, the error-free operation can both be achieved with 64- and 32-split after 75- and 100-km SMF transmission, respectively. At the OLT, each $10-\mathrm{Gb} / \mathrm{s}$ wavelength channel of the $40-\mathrm{Gb} / \mathrm{s}$ downstream signal has an average optical power of $3 \mathrm{dBm}$ (after wavelength multiplexed by the first WDM coupler). By considering the insertion losses of the circulator $=0.5 \mathrm{~dB}, 100-\mathrm{km}$ fiber loss $=20 \mathrm{~dB}$, typical $1 \times 64$ optical splitter $=20 \mathrm{~dB}$, and the second WDM coupler at the $\mathrm{ONU}=5 \mathrm{~dB}$. When the EDFA has a gain $=27 \mathrm{~dB}$ and the Rx sensitivity at BER of $10^{-9}=-22 \mathrm{dBm}$, the power budget of the 100-km reach system supporting 64 ONUs (each ONU has a symmetric downstream and upstream data rates of $40 \mathrm{~Gb} / \mathrm{s}$ ) is $6 \mathrm{~dB}$. In our measurement, as shown in Fig. 5, we can achieve 32 splits at $100-\mathrm{km}$ transmission. This is mainly limited by the signal-to-noise ratio of the signal. Although only the upstream signal was demonstrated, the downstream signal will have similar performances.

\section{CONCLUSION}

We have proposed and experimentally demonstrated a network architecture to achieve 40-Gb/s TDM-PON by employing four WDM $10-\mathrm{Gb} / \mathrm{s}$ externally OOK modulation channels. The transmission and the split-ratio of our proposed scheme have been analyzed. Error-free operation can be observed under 75and 100-km SMF transmission with 64 and 32 split-ratios, respectively, without dispersion compensation. We believe that the proposed architecture could be a promising candidate for next-generation $40-\mathrm{Gb} / \mathrm{s}$ TDM-PON.

\section{REFERENCES}

[1] Broadband Optical Access Systems Based on Passive Optical Network (PON), Recommendation G. 983.1, ITU-T, 1998.

[2] Gigabit-Capable Passive Optical Network (GPON): General Characteristics, Recommendation G. 984.1, ITU-T, 2003.

[3] Ethernet in the First Mile Task Force, IEEE 802.3ah, Draft 3.0b, 2004.

[4] D. Kettler, H. Kafka, and D. Spears, "Driving fiber to the home," IEEE Commun. Mag., vol. 38, no. 11, pp. 106-110, Nov. 2000.

[5] F. J. Effenberger, H. Ichibangase, and H. Yamashita, "Advances in broadband passive optical networking technologies," IEEE Commun. Mag., vol. 39, no. 12, pp. 118-124, Dec. 2001

[6] C. H. Yeh and C. W. Chow, "Utilization of four WDM channels with signal remodulation of OFDM-QAM for $10-\mathrm{Gb} / \mathrm{s}$ uplink passive optical networks," Opt. Commun., vol. 282, pp. 3701-3705, 2009.

[7] E. B. Desurvire, "Capacity demand and technology challenges for lightwave systems in the next two decades," J. Lightw. Technol., vol. 24, no. 12, pp. 4697-4710, Dec. 2006.

[8] R. E. Wagner, J. R. Igel, R. Whitman, M. D. Vaughn, A. B. Ruffin, and S. Bickham, "Fiber-based broadband-access deployment in the United States," J. Lightw. Technol., vol. 24, no. 12, pp. 4526-4540, Dec. 2006.

[9] 10 G EPON Study Group Public Articles [Online]. Available: http:// www.ieee802.org/3/av

[10] C. W. Chow, C. H. Yeh, C. H. Wang, F. Y. Shih, C. L. Pan, and S. Chi, "WDM extended reach passive optical networks using OFDM-QAM," Opt. Express, vol. 16, pp. 12096-12101, 2008.

[11] C. W. Chow, C. H. Yeh, C. H. Wang, F. Y. Shih, and S. Chi, "Signal remodulation of OFDM-QAM for long reach carrier distributed passive optical networks," IEEE Photon. Technol. Lett., vol. 21, no. 11, pp. 715-717, Jun. 1, 2009.

[12] C. W. Chow, G. Talli, A. D. Ellis, and P. D. Townsend, "Rayleigh noise mitigation in DWDM LR-PONs using carrier suppressed subcarrieramplitude modulated phase shift keying," Opt. Express, vol. 16, pp. 1860-1866, 2008.

[13] G. Talli, C. W. Chow, E. K. MacHale, and P. D. Townsend, "Rayleigh noise mitigation in long-reach hybrid DWDM-TDM PONs," J. Opt. Netw., vol. 6, pp. 765-776, 2007. 